\title{
Synchronization and desynchronization in the Olami-Feder-Christensen earthquake model and potential implications for real seismicity
}

\author{
S. Hergarten ${ }^{1,2}$ and R. Krenn ${ }^{1}$ \\ ${ }^{1}$ Institut für Erdwissenschaften, Karl-Franzens-Universität Graz, Heinrichstraße 26, 8010 Graz, Austria \\ ${ }^{2}$ Institut für Angewandte Geowissenschaften, Technische Universität Graz, Rechbauerstraße 12, 8010 Graz, Austria
}

Received: 17 June 2011 - Revised: 21 September 2011 - Accepted: 22 September 2011 - Published: 27 September 2011

\begin{abstract}
The Olami-Feder-Christensen model is probably the most studied model in the context of self-organized criticality and reproduces several statistical properties of real earthquakes. We investigate and explain synchronization and desynchronization of earthquakes in this model in the nonconservative regime and its relevance for the power-law distribution of the event sizes (Gutenberg-Richter law) and for temporal clustering of earthquakes. The power-law distribution emerges from synchronization, and its scaling exponent can be derived as $\tau=1.775$ from the scaling properties of the rupture areas' perimeter. In contrast, the occurrence of foreshocks and aftershocks according to Omori's law is closely related to desynchronization. This mechanism of foreshock and aftershock generation differs strongly from the widespread idea of spontaneous triggering and gives an idea why some even large earthquakes are not preceded by any foreshocks in nature.
\end{abstract}

\section{The Olami-Feder-Christensen model}

The Olami-Feder-Christensen (OFC) model (Olami et al., 1992) is a two-dimensional coupled map lattice model based on the Burridge-Knopoff spring-block earthquake model (Burridge and Knopoff, 1967). The model has attracted much attention not only as a paradigm for nonconservative systems in the context of self-organized criticality (SOC) (Bak et al., 1987; Bak, 1996; Jensen, 1998). It was soon recognized (Olami et al., 1992; Olami and Christensen, 1992) that it reproduces the most important statistical property of real earthquakes, the Gutenberg-Richter law (Gutenberg and

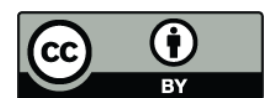

Correspondence to: S. Hergarten (stefan.hergarten@uni-graz.at)
Richter, 1954) (i.e., a power-law distribution of earthquake sizes) as well as the occurrence of large, more or less periodic earthquakes, so-called asperity events. Later it was discovered by Hergarten and Neugebauer (2002) that the OFC model also reproduces another fundamental property of earthquakes, the occurrence of foreshocks and aftershocks according to Omori's law (Omori, 1894; Utsu, 1961). In this context it was found that the foreshock and aftershock sequences of the model reproduce several further important statistical properties of real seismicity such as the increase of the number of foreshocks with the mainshock size and some kind of aftershock diffusion (Helmstetter et al., 2004).

Understanding how the OFC model generates earthquake sequences with apparently realistic statistical properties may finally help to understand the Gutenberg-Richter law, Omori's law and the occurrence of asperity-like events in nature. An idea on the origin of the power-law distribution was published rather early by Middleton and Tang (1995). Detailed studies on asperity events were recently published by Kawamura et al. (2010). In contrast, the occurrence of foreshocks and aftershocks in the OFC model still needs clarification.

The model itself refers to a square array of $L \times L$ blocks where each block is interconnected by springs with its four nearest neighbors and with a rigid driving plate moving at constant velocity. Each site $i$ supports a continuous variable $F_{i} \geq 0$ representing a force or local stress. The forces are initially set at random values, while the model itself is deterministic. The model is continuously driven by uniformly increasing all forces until any site $i$ achieves the threshold force $F_{i}=1$. In this case, the site $i$ becomes unstable and topples instantaneously into an equilibrium position $\left(F_{i}=0\right)$ and thereby transfers a fraction $\alpha$ of its force $F_{i}$ with $\alpha \in(0,0.25]$ to each of its four nearest neighbors. Now in turn one or more

Published by Copernicus Publications on behalf of the European Geosciences Union and the American Geophysical Union. 
of these neighbor sites may achieve or exceed the threshold force $\left(F_{i} \geq 1\right)$ and topple. This leads to an avalanche that continues until all sites are stable again $\left(F_{i}<1\right.$ for each $\left.i\right)$. Since the model is non-abelian, the relaxation rule must be applied in parallel to all sites that are simultaneously unstable.

The transmission parameter $\alpha$ settles the amount of $F_{i}$ that is dissipated during toppling since a fraction $4 \alpha$ of $F_{i}$ is transferred in total to the neighbors for sites in the bulk. Thus, total force is preserved during an event for $\alpha=0.25$, while the model is nonconservative for $\alpha<0.25$.

After a transient time, the system approaches an apparently critical state characterized by a power-law distribution of the event sizes. However, the emergence of criticality in the nonconservative regime has been debated from the very beginning (Olami et al., 1992; Grassberger, 1994; de Carvalho and Prado, 2000; Christensen et al., 2001; de Carvalho and Prado, 2001; Drossel, 2002; Miller and Boulter, 2002, 2003; Wissel and Drossel, 2006). In return, temporal correlations similar to those found in real seismicity (foreshocks, aftershocks, and asperity events) were only recognized in the nonconservative regime (Olami and Christensen, 1992; Hergarten, 2002; Helmstetter et al., 2004). We therefore focus on the nonconservative case in this study.

\section{The basic mechanism of synchronization}

Boundary conditions play a crucial role in the OFC model. It was soon recognized that the model does not display SOCbehavior under periodic boundary conditions (Socolar et al., 1993; Grassberger, 1994). The basic mechanism how sites synchronize from the boundaries to form larger events was first investigated by Middleton and Tang (1995). Without any boundary effects, all sites might topple periodically at a period $1-4 \alpha$ independently of each other. The period results from the fact that each time a site topples it drops one unit of force and receives a force $\alpha$ from each of its four neighbors. However, boundary sites receive less force from their neighbors, resulting in a lower toppling frequency. If one of the neighbors of a site $i$ does not topple within a time span $1-4 \alpha$, the site $i$ cannot topple and must wait until the delayed site $j$ topples and delivers its contribution $\alpha$. At this point, site $i$ becomes immediately unstable, i.e., it synchronizes with the site $j$. Middleton and Tang developed a simplified model involving the phases of toppling instead of the forces and numerically found power-law distributed event sizes.

Apart from its directed structure (as only synchronization in direction of two of the four neighbors is considered), the approach of Middleton and Tang differs from the OFC model by neglecting "supercritical" forces $F_{i}>1$. In the OFC model, only hypocenters always topple exactly at $F_{i}=1$, while all other sites involved in an event may topple at $F_{i}>1$.

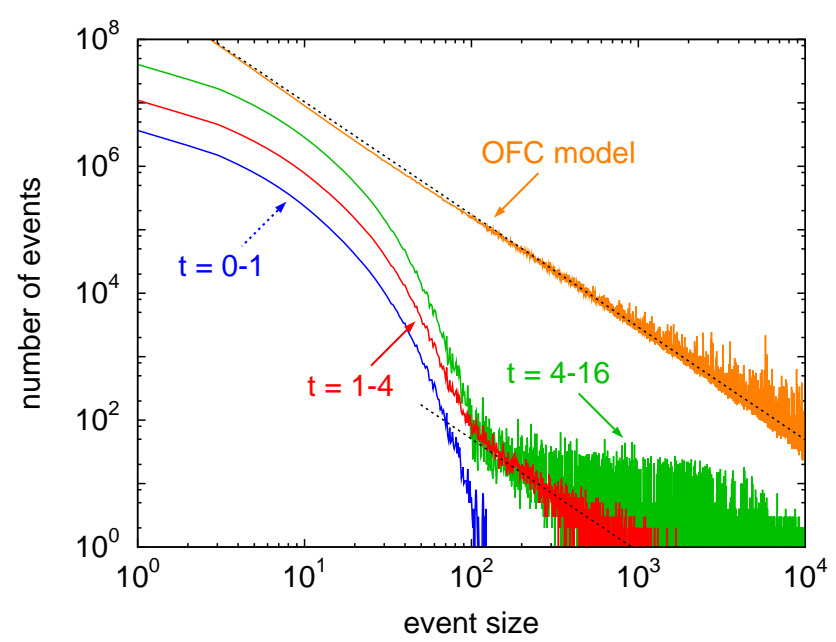

Fig. 1. Event-size statistics of the modified OFC model without supercritical forces measured over different time intervals on a $4096 \times 4096$ grid with $\alpha=0.2$ and random initial values. The orange line refers to $2 \times 10^{9}$ events in the quasi-steady state of the original OFC model. The dashed lines correspond to a power-law distribution with a scaling exponent $\tau=1.775$ that is discussed in Sect. 3.

In order to demonstrate the importance of these supercritical forces for the OFC model we investigate a modified version where supercritical forces are neglected, i.e., where each unstable site only transfers an amount $\alpha$ of force to its neighbors even if $F_{i}>1$. The rest is simply lost. We simulated this model with $\alpha=0.2$ on a $4096 \times 4096$ lattice with random initial conditions and found that it synchronizes from the boundaries as expected. Figure 1 shows the event-size statistics for different time intervals. In the time interval $t \in[1,4]$, the event-size distribution follows a power law for event sizes from about 100 to 1000 . The statistics of the smaller events are governed by the still unsynchronized events in the bulk. The scaling exponent of the power law matches that found for the original OFC model within the accuracy of the data. We verified that these results hold at least for $\alpha \in[0.16,0.24]$.

However, as synchronization proceeds towards the interior of the domain, larger events dominate, so that the power law distribution is soon lost. This happens long before synchronization reaches the middle of the domain. For $\alpha=0.2$, the transition occurs when the synchronized region has a width of less than 100 sites. Thus, criticality is only a transient phenomenon in the model without supercritical forces; it evolves rapidly towards a supercritical state. Therefore, selforganized criticality in the OFC model must be closely related to the occurrence of supercritical forces $F_{i}>1$, which will be discussed in Sect. 4 . 


\section{The scaling exponent of the event-size distribution}

We now derive a semi-phenomenological approach to explain the power-law distribution arising from synchronization. The idea is in its spirit identical to the hierarchical clustering approach recently suggested by Hergarten and Krenn (2011) to explain the dynamics of the Drossel-Schwabl forest-fire model (Drossel and Schwabl, 1992).

A cluster is here defined in the same way as in site percolation where two sites belong to the same cluster if there exists a continuous path of nearest neighbor sites connecting them. Following the ideas of the previous section, we assume that a cluster of synchronized sites persists until it is randomly captured via its hypocenter by another cluster which has experienced a delay in toppling. Let $N(s)$ be the number of clusters of size $s$ on a lattice of total size $A=L^{2}$. If another given cluster topples with a sufficient delay, it captures our cluster in case the hypocenter of our cluster is located on the accessible perimeter $p_{a}$ of the delayed cluster. The accessible perimeter of a cluster consists of those perimeter sites which can be reached, in principle, by a random walker coming from infinity (Grossman and Aharony, 1986), and it was recently used in a modified forest-fire model to mimic ignition by human impact (Krenn and Hergarten, 2009).

Assuming a random alignment of all clusters, our site is captured by the delayed cluster with a probability $\frac{p_{a}}{A}$. Capture of clusters can then in principle be described by Smoluchowski's coagulation equation with a suitable kernel. Although capture is in principle possible among clusters of any sizes, its effect on the cluster size is largest if both clusters are of similar sizes. We therefore only consider the case that one size is not larger than twice the other size, i.e., that two clusters with sizes in the interval $\left[\frac{1}{2} \sqrt{2} s, \sqrt{2} s\right]$ turn into one cluster with a size between $\sqrt{2} s$ and $2 \sqrt{2} s$. As the number of clusters in this interval is approximately $\sqrt{\frac{1}{2}} s N(s)$, the number of pairs is $\frac{1}{2}\left(\sqrt{\frac{1}{2}} s N(s)\right)^{2}$, so that the number of capture events (caused by delay of one cluster) is roughly $\frac{1}{2}\left(\sqrt{\frac{1}{2}} s N(s)\right)^{2}\left(\frac{p_{a}(s)}{A}\right)$. Thus, the number of clusters in the interval $\left[\frac{1}{2} \sqrt{2} s, \sqrt{2} s\right]$ changes by

$$
\begin{aligned}
\sqrt{\frac{1}{2}} s \delta N(s) & =\frac{1}{2}\left(\sqrt{\frac{1}{2}} \frac{s}{2} N\left(\frac{s}{2}\right)\right)^{2} \frac{p_{a}\left(\frac{s}{2}\right)}{A} \\
& -2 \frac{1}{2}\left(\sqrt{\frac{1}{2}} s N(s)\right)^{2} \frac{p_{a}(s)}{A},
\end{aligned}
$$

where the factor 2 in the second line states that two clusters vanish in a single capture event. In equilibrium, which can only be achieved if a sufficient number of clusters of size one is supplied, $\delta N(s)$ must vanish. This leads to

$$
\frac{1}{2} p_{a}\left(\frac{s}{2}\right)\left(\frac{s}{2} N\left(\frac{s}{2}\right)\right)^{2}=p_{a}(s)(s N(s))^{2},
$$

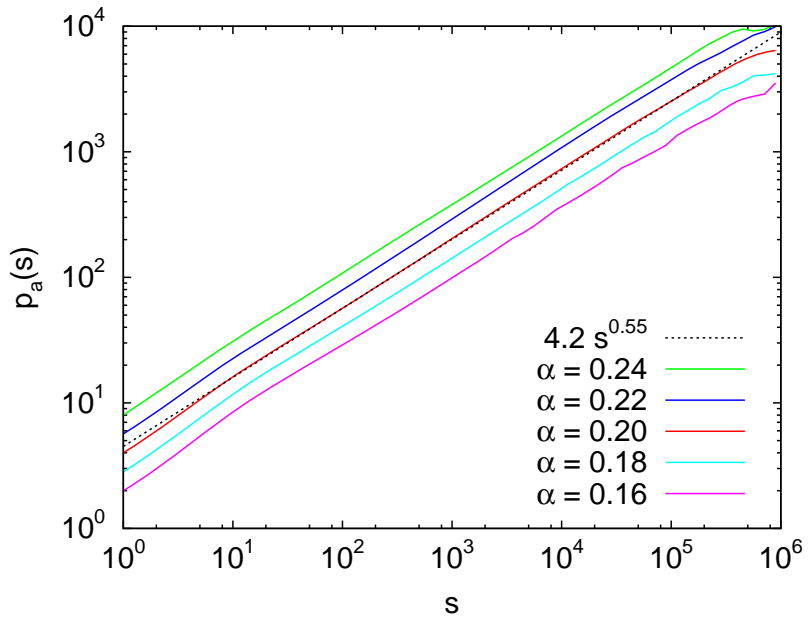

Fig. 2. Scaling behavior of the accessible perimeter of events in the OFC model numerically determined for different values of $\alpha$ on a $1024 \times 1024$ lattice. The curves referring to $\alpha \neq 0.20$ were shifted vertically for clarity.

so that

$N(s) \sim s^{-\frac{3}{2}} p_{a}(s)^{-\frac{1}{2}}$.

Figure 2 shows the accessible perimeter of clusters in the OFC model derived from numerical simulations on a $1024 \times$ 1024 lattice. The results suggest that $p_{a}(s)$ scales like

$p_{a}(s) \sim s^{h}$

with $h=0.55$ independently of $\alpha$ at least for $0.16 \leq \alpha \leq 0.24$. This immediately leads to

$N(s) \sim s^{-\tau}$

with a scaling exponent $\tau=1.775$ in perfect agreement with the numerical results presented in Fig. 1.

\section{Desynchronization by supercritical forces}

We now extend the idea of stress concentration proposed by Kawamura et al. (2010). They observed that the forces of the toppling sites approach one during a sequence of asperity events and provided a first explanation of this phenomenon.

Let us first come back to the basic mechanism of synchronization. Due to a delay in the toppling of a site $j$, the (neighbored) site $i$ cannot topple before $j$ topples, so that they topple simultaneously. Then, the site $i$ topples at a force $F_{i}=1+\tau$ instead of $F_{i}=1$ where $\tau$ is the delay the site $i$ experiences. Therefore, it transfers an amount $\alpha(1+\tau)$ instead of $\alpha$ to site $j$, so that this site will topple earlier next time, i.e., after a time span $T=1-4 \alpha-\alpha \tau$. At this time, the force at the site $i$ will be $F_{i}=1-\alpha \tau<1$ including the contribution 


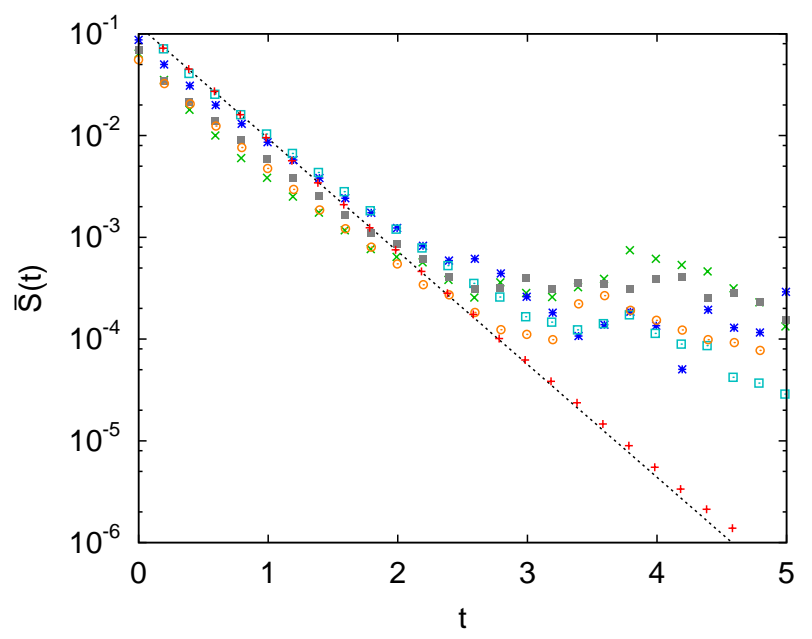

Fig. 3. Decay of the mean supercritical force excess $\bar{S}$ in six sequences of asperity events for $\alpha=0.2$. The dashed line corresponds to the theoretical decay according to $\bar{S}(t) \sim \exp (-2.55 t)$.

of site $j$, so that it will not topple simultaneously with $j$, but delayed by $\tau^{\prime}=\alpha \tau$. So the synchronization of the two sites is immediately lost, but they will topple with a short time lag in future. As $\tau \leq \alpha$ in most cases, $\tau^{\prime} \leq \alpha^{2}$, which means that the time lag will be shorter for small $\alpha$. As a consequence of the small time lag, even a small delay in the toppling of site $j$ will be sufficient to resynchronize the sites temporarily.

In principle, the same mechanism applies to larger events, although they are not broken immediately. Let us consider an event where $h$ is the hypocenter, $E$ is the set of all involved sites, $s$ is the size, $N(i)$ is the neighborhood of the site $i$, and $F_{i}$ is the (supercritical) force of site $i$ when it topples. The hypocenter will topple next time after a time span

$T=1-\alpha \sum_{j \in N(h)} F_{j}$

Including the contributions from its neighbors, each site $i \in$ $E$ will have a force

$F_{i}^{\prime}=T+\alpha \sum_{j \in N(i)} F_{j}$,

so that the supercritical force excess $S_{i}=F_{i}-1$ of the site $i$ turns into

$S_{i}^{\prime}=\alpha\left(\sum_{j \in N(i)} S_{j}-\sum_{j \in N(h)} S_{j}\right)$.

As long as $S_{i}^{\prime} \geq 0$ for all $i \in E$, the event will take place (at least) at the same size. If this happens several times, a perfect sequence of asperity events arises. In return, if $S_{i}^{\prime}<0$ for any site, the event will cease at site $i$. This site becomes the hypocenter of a new event which takes place with a probably small delay $\left|S_{i}^{\prime}\right|$. Thus, the event is broken into two or even more events.

The first sum in Eq. (8) describes a diffusive decay of the values $S_{i}$, while the second sum can be seen as an inhomogeneity that may cause negative values and thus desynchronization. The decay of the mean supercritical force excess $\bar{S}$ can be estimated by assuming that $E$ is surrounded by events without any supercritical force (as it is the case for events of size one), i.e., that $S_{i}=0$ for $i \notin E$. In this case, Eq. (8) yields

$$
\begin{aligned}
\bar{S}^{\prime} & =\alpha\left(\frac{1}{s} \sum_{i \in E} \sum_{j \in N(i)} S_{j}-\sum_{j \in N(h)} S_{j}\right) \\
& =\alpha\left(\frac{1}{s} \sum_{i \in E} n_{i} S_{i}-\sum_{j \in N(h)} S_{j}\right)
\end{aligned}
$$

where $n_{i}$ is the internal coordination number of the site $i$, i. e., the number of its neighbors which belong to $E$. As clusters of sites toppling simultaneously are rather compact in the OFC model, almost all sites involved in a large event should have $n_{i}=4$, so that

$$
\bar{S}^{\prime} \approx \alpha\left(4 \bar{S}-\sum_{j \in N(h)} S_{j}\right) .
$$

If the force excess in $N(h)$ is the same as $\bar{S}$, too, we immediately obtain

$\bar{S}^{\prime} \approx \alpha\left(4-n_{h}\right) \bar{S}$.

As the decay from $\bar{S}$ to $\bar{S}^{\prime}$ takes place within a time span of length $1-4 \alpha$ (strictly speaking, slightly shorter), the mean force excess of an event sequence decays exponentially according to

$\bar{S}(t) \sim \exp (-\lambda t)$

with

$\lambda=-\frac{\log \left(\left(4-n_{h}\right) \alpha\right)}{1-4 \alpha}$.

Obviously, events where the hypocenter is a "nose" at the border of the event $\left(n_{h}=1\right)$ have the lowest decay constant, so that they shall be most stable against decay, followed by those with hypocenters at a corner $\left(n_{h}=2\right)$. This result gives an explanation for the recent finding that the vast majority of the asperity events has $n_{h}=1$, followed by $n_{h}=2$ (Kawamura et al., 2010). In return, Eq. (8) immediately reveals that desynchronization is more likely at the borders where $n_{i}<4$. In particular, noses which are not hypocenters will rapidly lose synchronization, leading to rather smooth borders of asperity events.

The data shown in Fig. 3 confirm our result on the exponential decay numerically for $\alpha=0.2$ by tracing the mean 


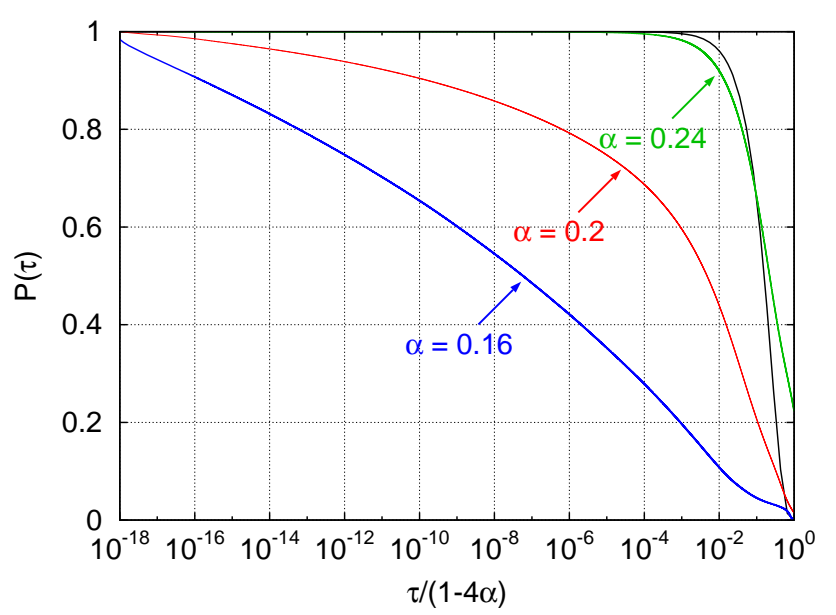

Fig. 4. Cumulative distribution $P(\tau)$ of the time spans $\tau$ between receiving the last portion of force from a neighbor before becoming unstable and time of instability. The black line shows the distribution that would arise from completely uncorrelated events.

supercritical forces of large asperity events. Six sequences where the hypocenter persisted for at least 25 periods were selected. According to Eq. (14), the smallest decay constant should be $\lambda=2.55$ here. One of these 6 sequences obeys the exponential decay even down to $\bar{S}(t) \approx 10^{-6}$, while the majority of sequences tends to larger supercritical forces when $\bar{S}(t) \approx 10^{-3}$, presumably caused by the supply of supercritical forces from other events in the neighborhood. In some cases, the decay is slightly slower than predicted by Eqs. (13) and (14). This occurs if the supercritical forces in the neighborhood of the hypocenter is lower than the mean supercritical force of the whole event.

\section{Temporal correlations}

As discussed in the previous section, large events tend to be broken into sequences of smaller events with a small time lag. This obviously causes a strong short-term correlation of events. In Fig. 4, the time span $\tau$ between receiving the last portion of force from a neighbor before becoming unstable and time of instability is analyzed for all hypocenters. The distributions were derived from simulations on a $2048 \times 2048$ grid using an implementation where the forces are stored in 64 bit integer format with a numerical precision of $\epsilon=2^{-62} \approx 2 \times 10^{-19}$ (Krenn and Hergarten, 2011).

If the events were completely uncorrelated, and each of the four neighbors topples once per time span $1-4 \alpha$, the probability should be $P(\tau)=\left(1-\frac{\tau}{1-4 \alpha}\right)^{4}$. The OFC model apparently approaches this distribution for $\alpha \rightarrow 0.25$, while the correlations become stronger as $\alpha$ decreases. For $\alpha=0.16$ even values $\tau$ down to the numerical precision occur with significant frequency. This phenomenon is interesting for

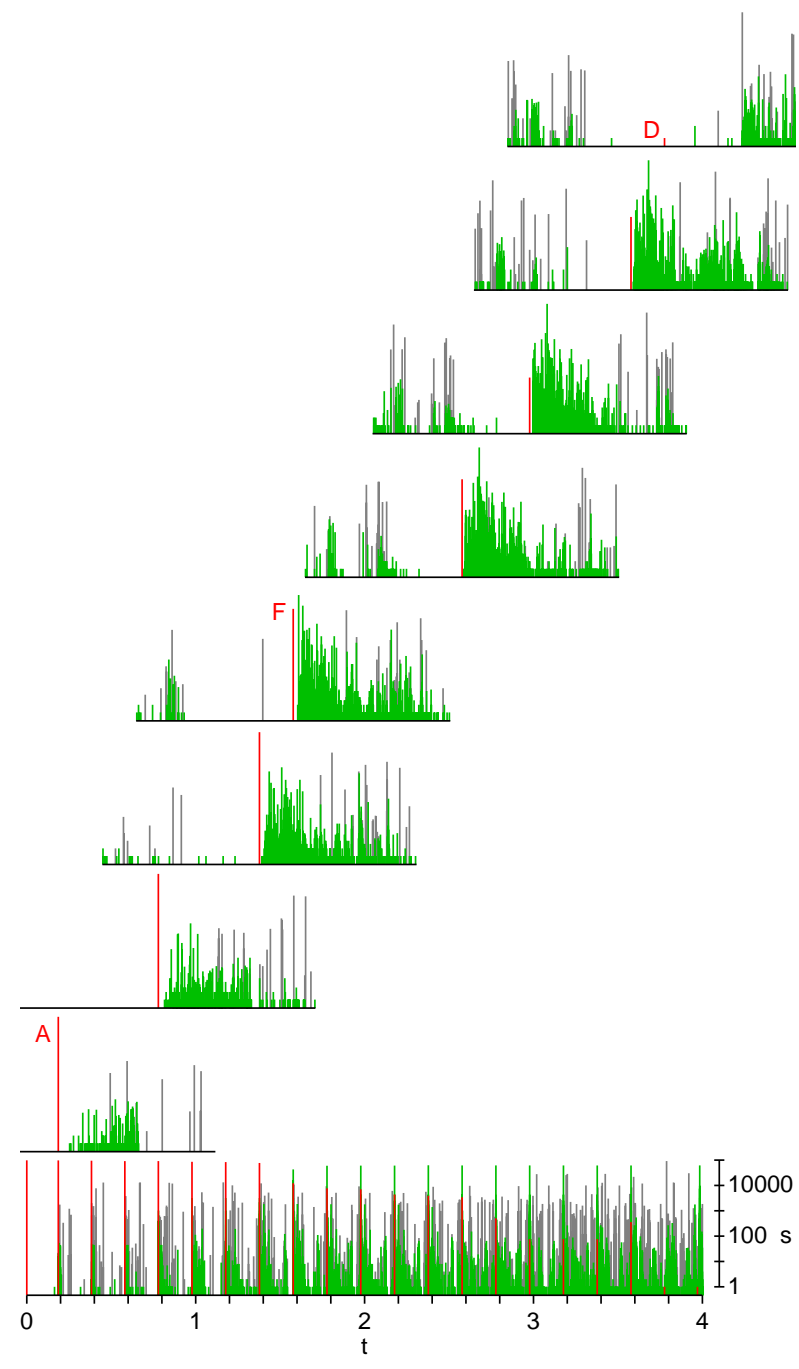

Fig. 5. A sequence of events computed on a $512 \times 512$ grid, starting from a large event spanning $37 \%$ of the grid. Only events overlapping the rupture area of the initial event are plotted. Red bars: Events starting from the same hypocenter as the first one. Green bars: Events starting from hypocenters within the rupture area of the first event. Grey bars: Events starting from outside the rupture area of the first event. The upper plots are parts of the sequence around selected events where the time axis is nonlinearly stretched according to $t_{\text {stretched }}=t_{\text {redbar }}+2 \sqrt[3]{t-t_{\text {redbar }}}$.

the behavior of the OFC model in the limit of infinite precision (Drossel, 2002; Miller and Boulter, 2003), but has no meaning with respect to real earthquakes: The propagation of a large earthquake takes at least several seconds in nature, so that the minimum detectable values $\tau$ are much lower than the numerical precision used here (Helmstetter et al., 2004; Hergarten and Jansen, 2005).

The mechanism of desynchronization with very small time lags generates sequences of foreshocks, a mainshock and aftershocks, where the mainshock is simply the largest event 


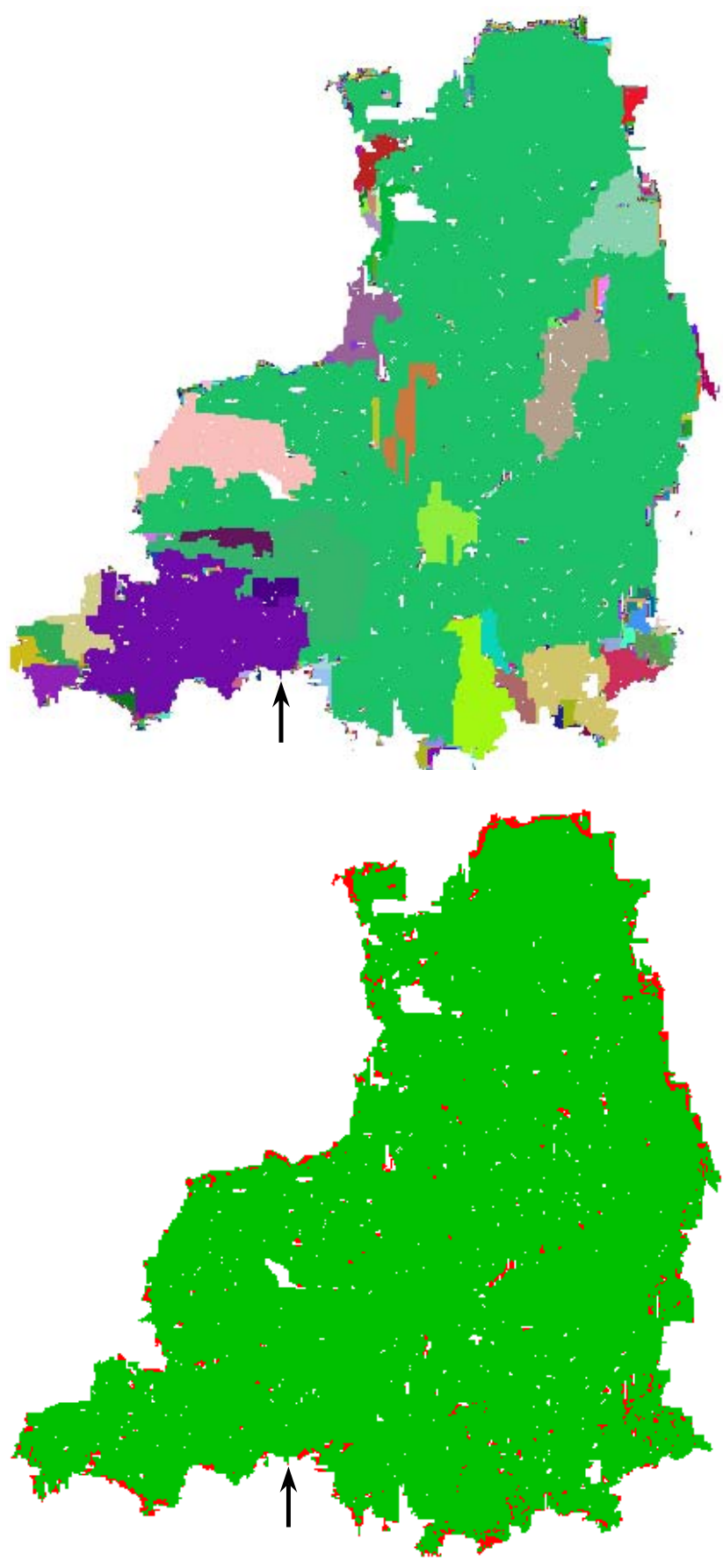

Fig. 6. Top: State of desynchronization of the first event from Fig. 5 at $t=3$. Individual events are marked with random colors. Bottom: Respective hypocenters (red points). The arrow marks the hypocenter of the initial event.

according to the usual definition. The conjecture that foreshocks and aftershocks arise from desynchronization of large earthquakes is somewhat opposite to the widespread idea of spontaneous triggering as it is, e.g., assumed in the epidemic type aftershock model (Ogata, 1988).
Figure 5 shows an example of a decaying asperity sequence. The second (marked with an A) occurrence is already accompanied by aftershocks, and their number and size increases in the following. Most notably, no foreshocks are observed at this stage. The 9th occurrence (marked with a F) is the first one where the initial event (red) is no longer the largest one, so that it will be no longer the mainshock, but a foreshock of a larger event. At event no. 20 (marked with a D), the asperity has been destroyed; almost the entire initial rupture area has been captured by an event with a hypocenter outside this area with a significant time lag.

Figure 6 shows how the initial event has been broken at $t=3$ and the respective hypocenters. The hypocenters accumulate at the border of the initial earthquake indicating that desynchronization preferably takes place there. This phenomenon was already explained above from Eqs. (8) and (10). Furthermore it immediately explains why aftershocks are preferred to foreshocks.

\section{Omori's law}

In the following, we present a simple idea of fragmentation to explain the origin of Omori's law based on desynchronization. Let us start with one event involving $s_{0} \gg 1$ sites at time $t_{0}$. In the first step, this event falls into two pieces of size $q s_{0}$ and $(1-q) s_{0}$ with a delay $\tau$ in time. For simplicity we assume that the first one is still at time $t_{0}$ (modulo the period), so that the second takes place at $t_{0}+\tau$. In the next step, each event falls into two pieces according to the same mechanism, but with a smaller delay $\frac{1}{2} \tau$. This factor is motivated by the exponential decay of the supercritical forces discussed above. If we repeat this procedure infinitely, we recognize that $q^{n} s_{0}$ sites topple at a total delay smaller than $\left(\frac{1}{2}\right)^{n} \tau$. Thus, the number of sites that topple with a delay smaller than $t$ is

$s(t)=s_{0} q^{-\log _{2} \frac{t-t_{0}}{\tau}}=s_{0}\left(\frac{t-t_{0}}{\tau}\right)^{-\log _{2} q}$,

so that the number of toppling sites per time is

$\dot{s}(t) \sim\left(t-t_{0}\right)^{-\left(1+\log _{2} q\right)}$.

This power law decay does not describe the number of aftershocks per time directly like Omori's law, but a mixture of number and event size. However, if we assume that all aftershocks obey the same size distribution (i.e., the GutenbergRichter law), it turns into Omori's law with an exponent $p=\left(1+\log _{2} q\right)<1$. If $q$ approaches 1 (i.e., if desynchronization is strongly confined to the border), $p$ approaches 1. At this point it should be mentioned that it was already observed that the OFC model yields $p<1$ (Hergarten and Neugebauer, 2002; Helmstetter et al., 2004), while values $p>1$ are frequently found in nature (Kisslinger and Jones, 
1991). Values $p>1$ were also observed in modified versions of the OFC model (Hainzl et al., 1999; Jagla, 2010).

It is noteworthy, too, that this mechanism of aftershock generation hinges on a stepwise desynchronization during a sequence of asperity events. Disturbances in the force transfer close to the hypocenters will affect the small time lags between the aftershocks which may result in resynchronization and thus wipe out Omori's law. This explains an observation made by Yamamoto et al. (2010) who found numerically that Omori's law is destroyed even by a very small dynamic randomness in the force transfer, while the Gutenberg-Richter law and the occurrence of asperity events itself is more robust.

\section{Conclusions and potential implications for real seismicity}

We found that the dynamics of the OFC model is governed by two competing mechanisms: Synchronization pushes the system towards a critical state and thus generates the Gutenberg-Richter law. In return, desynchronization prevents the system from becoming overcritical and generates foreshocks and aftershocks.

The theoretical concept proposed in Sect. 3 suggests that the scaling exponent of the event-size distribution is $\tau=$ 1.775 , independently of the parameter $\alpha$, as long as synchronization is sufficiently strong. However, the concept does not allow an estimate of the minimum value of $\alpha$ that is sufficient to maintain synchronization. Desynchronization may disturb the power-law distribution, so that our results suggest that any dependency of $\tau$ on $\alpha$ is a spurious effect.

In contrast, a dependence on Richter's b-value (which is closely related to $\tau$ ) on the type of fault zones was recently found in real seismicity (Narteau et al., 2009). However, this result is not in contradiction to our findings since numerical simulations of the OFC model for different values of $\alpha$ and different lattice sizes practically yield a variation in $\tau$.

The mechanism of foreshock and aftershock generation by desynchronization differs in its spirit strongly from the widespread idea of spontaneous triggering of aftershocks. If foreshocks and aftershocks are the result of desynchronization, a "new" (i.e., just generated by synchronization) asperity event should occur without foreshocks and with only few aftershocks. As synchronization hinges on a delayed occurrence, there should be an increased probability of a large event without foreshocks if the time since the last earthquake is longer than the characteristic asperity recurrence time. Furthermore, the "productivity", i.e., the number of aftershocks (and foreshocks) would mainly depend on the "age" of the asperity (beside the mainshock magnitude). This may be the reason for the large and apparently random variability of the number of foreshocks and aftershocks found in nature.
Even more important, our approach also provides a simple explanation of Omori's law which is one of the most fundamental relationships in statistical seismology. Beyond this, the mechanism of desynchronization immediately explains another phenomenon sometimes found in real earthquake sequences: Foreshocks migrate towards the hypocenter of the mainshock (Kagan and Knopoff, 1978; von Seggern et al., 1981), and aftershocks diffuse from the hypocenter of the mainshock (Tajima and Kanamori, 1985; Marsan et al., 2000).

Although our results provide several arguments in favor of desynchronization being responsible for foreshocks and aftershocks, it currently seems to be impossible to decide whether real foreshocks and aftershocks arise from this mechanism or not as there are still too many open questions concerning the nature of asperity events. One may argue that the OFC model is too simple and obviously contains unrealistic simplifications such as a constant loading rate at all sites. Variations in the loading rate would weaken synchronization, but this would mainly cause a faster decay of the asperities and thus more foreshocks and aftershocks. This would even be reasonable since it was already found that the number of foreshocks and aftershocks in the OFC model is too low compared to nature (Helmstetter et al., 2004). In contrast, the arguments that our simple mechanism of fragmentation only predicts Omori exponents $p<1$ and is not robust against random disturbances cannot be refuted so far, leading to the conclusion that this mechanism is probably not the only one responsible for aftershocks in real seismicity.

Acknowledgements. This work was funded by the Austrian Science Fund (FWF): P19733-N10.

Edited by: L. Telesca

Reviewed by: two anonymous referees

\section{References}

Bak, P.: How Nature Works - the Science of Self-Organized Criticality, Copernicus, Springer, Berlin, Heidelberg, New York, 1996.

Bak, P., Tang, C., and Wiesenfeld, K.: Self-organized criticality. An explanation of 1/f noise, Phys. Rev. Lett., 59, 381-384, 1987.

Burridge, R. and Knopoff, L.: Model and theoretical seismicity, Bull. Seismol. Soc. Am., 57, 341-371, 1967.

Christensen, K., Hamon, D., Jensen, H. J., and Lise, S.: Comment on "Self-organized criticality in the Olami-Feder-Christensen model, Phys. Rev. Lett., 87, 039 081, 2001.

de Carvalho, J. X. and Prado, C. P. C.: Self-organized criticality in the Olami-Feder-Christensen model, Phys. Rev. Lett., 84, 40064009, 2000.

de Carvalho, J. X. and Prado, C. P. C.: Reply to comment on Self-organized criticality in the Olami-Feder-Christensen model, Phys. Rev. Lett., 87, 039082, doi:10.1103/PhysRevLett. 87.039802, 2001. 
Drossel, B.: Complex scaling behavior in nonconserved selforganized critical systems, Phys. Rev. Lett., 89, 238701, doi: 10.1103/PhysRevLett.89.238701, 2002.

Drossel, B. and Schwabl, F.: Self-organized critical forest-fire model, Phys. Rev. Lett., 69, 1629-1632, 1992.

Grassberger, P.: Efficient large-scale simulations of a uniformly driven system, Phys. Rev. E, 49, 2436-2444, 1994.

Grossman, T. and Aharony, A.: Structure and perimeters of percolation clusters, J. Phys. A: Math. Gen., 19, L745-L751, doi:10.1088/0305-4470/19/12/009, 1986.

Gutenberg, B. and Richter, C. F.: Seismicity of the Earth and Associated Phenomenon., Princeton University Press, Princeton, 2nd edn., 1954.

Hainzl, S., Zöller, G., and Kurths, J.: Similar power laws for foreshock and aftershock sequences in a spring-block model for earthquakes, J. Geophys. Res., 104, 7243-7253, 1999.

Helmstetter, A., Hergarten, S., and Sornette, D.: Properties of foreshocks and aftershocks of the nonconservative self-organized critical Olami-Feder-Christensen model, Phys. Rev. E, 70, 046120, doi:10.1103/PhysRevE.70.046120, 2004.

Hergarten, S.: Self-Organized Criticality in Earth Systems, Springer, Berlin, Heidelberg, New York, 2002.

Hergarten, S. and Jansen, F.: On the separation of timescales in spring-block earthquake models, Nonlin. Processes Geophys., 12, 83-88, doi:10.5194/npg-12-83-2005, 2005.

Hergarten, S. and Krenn, R.: A semi-phenomenological approach to explain the event-size distribution of the Drossel-Schwabl forestfire model, Nonlin., Processes Geophys., 18, 381-388, doi:10. 5194/npg-18-381-2011, 2011.

Hergarten, S. and Neugebauer, H. J.: Foreshocks and aftershocks in the Olami-Feder-Christensen model, Phys. Rev. Lett., 88, 238501, 2002.

Jagla, E. A.: Realistic spatial and temporal earthquake distributions in a modified Olami-Feder-Christensen model, Phys. Rev. E, 81, 046117, doi:10.1103/PhysRevE.81.046117, 2010.

Jensen, H. J.: Self-Organized Criticality - Emergent Complex Behaviour in Physical and Biological Systems, Cambridge University Press, Cambridge, New York, Melbourne, 1998.

Kagan, Y. Y. and Knopoff, L.: Statistical study of the occurrence of shallow earthquakes, Geophys. J. R. Astron. Soc., 55, 67-86, 1978.

Kawamura, H., Yamamoto, T., Kotani, T., and Yoshino, H.: Asperity characteristics of the Olami-Feder-Christensen model of earthquakes, Phys. Rev. E, 81, 031 119, doi:10.1103/PhysRevE. 81.031119, 2010.

Kisslinger, C. and Jones, L. M.: Properties of aftershock sequences in southern California, J. Geophys. Res., 96, 11947-11958, 1991.

Krenn, R. and Hergarten, S.: Cellular automaton modelling of lightning-induced and man made forest fires, Nat. Hazards Earth Syst. Sci., 9, 1743-1748, doi:10.5194/nhess-9-1743-2009, 2009.
Krenn, R. and Hergarten, S.: An efficient integer algorithm for the Olami-Feder-Christensen model, In preparation, 2011.

Marsan, D., Bean, C. J., Steacy, S., and McCloskey, J.: Observation of diffusion processes in earthquake populations, and implications for the predictability of seismicity systems, J. Geophys. Res., 105, 28081-28094, 2000.

Middleton, A. A. and Tang, C.: Self-organized criticality in nonconserved systems, Phys. Rev. Lett., 74, 742-745, 1995.

Miller, G. and Boulter, C. J.: Measurements of criticality in the Olami-Feder-Christensen model, Phys. Rev. E, 66, 016123, doi:10.1103/PhysRevE.66.016123, 2002.

Miller, G. and Boulter, C. J.: Crossover behavior in the event size distribution of the Olami-Feder-Christensen model, Phys. Rev. E, 67, 046 114, doi:10.1103/PhysRevE.67.046114, 2003.

Narteau, C., Byrdina, S., Shebalin, P., and Schorlemmer, D.: Common dependence on stress for the two fundamental laws of statistical seismology, Nature, 462, 642-645, doi:10.1038/nature08553, 2009.

Ogata, Y.: Statistical models for earthquake occurrences and residual analysis for point processes, J. Am. Stat. Assoc., 83, 9-27, 1988.

Olami, Z. and Christensen, K.: Temporal correlations, universality, and multifractality in a spring-block model of earthquakes, Phys Rev. A, 46, R1720-R1723, 1992.

Olami, Z., Feder, H. J. S., and Christensen, K.: Self-organized criticality in a continuous, nonconservative cellular automation modeling earthquakes, Phys. Rev. Lett., 68, 1244-1247, 1992.

Omori, F.: On the aftershocks of earthquakes, J. Coll. Sci. Imp. Univ. Tokyo, 7, 111-200, 1894.

Socolar, J. E. S., Grinstein, G., and Jayaprakash, C.: On selforganized criticality in nonconserving systems, Phys. Rev. E, 47, 2366-2376, doi:10.1103/PhysRevE.47.2366, 1993.

Tajima, F. and Kanamori, H.: Global survey of aftershock area expansion patterns, Phys. Earth Planet. Inter., 40, 77-134, 1985.

Utsu, T.: A statistical study on the occurrence of aftershocks, Geophys. Mag., 30, 521-605, 1961.

von Seggern, D., Alexander, S. S., and Baag, C.-E.: Seismicity parameters preciding moderate to major earthquakes, J. Geophys. Res., 86, 9325-9351, doi:10.1029/JB086iB10p09325, 1981.

Wissel, F. and Drossel, B.: Transient and stationary behavior of the Olami-Feder-Christensen model, Phys. Rev. E, 74, 066109, doi:10.1103/PhysRevE.74.066109, 2006.

Yamamoto, T., Yoshino, H., and Kawamura, H.: Simulation study of the inhomogeneous Olami-Feder-Christensen model of earthquakes, Eur. Phys. J. B, 77, 559-564, 2010. 